INPLASY

PROTOCOL

To cite: Wang et al. A systematic review and network meta-analysis of single nucleotide polymorphisms associated with breast cancer risk. Inplasy protocol

202220010. doi:

10.37766/inplasy2022.2.0010

Received: 06 February 2022

Published: 06 February 2022

Corresponding author:

Ying yuan Wang

wyy15596828515@163.com

Author Affiliation:

Xiangya Medical College, Central South University,

Changsha, Hunan.

Support: Xiangya Medical College.

Review Stage at time of this submission: Data analysis.

Conflicts of interest:

None declared.

\section{A systematic review and network meta-analysis of single nucleotide polymorphisms associated with breast cancer risk}

\author{
Wang, YY1; Chen, ZC2; Zhang, LX3; Chen, SY4.
}

Review question / Objective: P: Breast cancer patient; I: Single nucleotide polymorphisms associated with breast cancer risk; C: Healthy person; O: By comparing the proportion of SNP mutations in the tumor group and the control group, the effect of BREAST cancer risk-related SNP was investigated; S: Case-control study.

Condition being studied: Breast cancer (BC) is one of the most common cancers among women, and its morbidity and mortality have continued to increase worldwide in recent years, reflecting the strong invasiveness and metastasis characteristics of this cancer. BC is a complex disease that involves a sequence of genetic, epigenetic, and phenotypic changes. Polymorphisms of genes involved in multiple biological pathways have been identified as potential risks of BC. These genetic polymorphisms further lead to differences in disease susceptibility and severity among individuals. The development of accurate molecular diagnoses and biological indicators of prognosis are crucial for individualized and precise treatment of $\mathrm{BC}$ patients.

INPLASY registration number: This protocol was registered with the International Platform of Registered Systematic Review and Meta-Analysis Protocols (INPLASY) on 06 February 2022 and was last updated on 06 February 2022 (registration number INPLASY202220010).

\section{INTRODUCTION}

Review question / Objective: P: Breast cancer patient; I: Single nucleotide polymorphisms associated with breast cancer risk; C: Healthy person; O: By comparing the proportion of SNP mutations in the tumor group and the control group, the effect of BREAST cancer risk-related SNP was investigated; S: Casecontrol study.

Condition being studied: Breast cancer (BC) is one of the most common cancers among women, and its morbidity and mortality have continued to increase worldwide in recent years, reflecting the strong invasiveness and metastasis 
characteristics of this cancer. $B C$ is a complex disease that involves a sequence of genetic, epigenetic, and phenotypic changes. Polymorphisms of genes involved in multiple biological pathways have been identified as potential risks of $\mathrm{BC}$. These genetic polymorphisms further lead to differences in disease susceptibility and severity among individuals. The development of accurate molecular diagnoses and biological indicators of prognosis are crucial for individualized and precise treatment of $\mathrm{BC}$ patients.

\section{METHODS}

Search strategy: Database: Pubmed and Embase $((()((($ "Polymorphism, Single Nucleotide"[Mesh]) OR (Nucleotide Polymorphism, Single[Title/Abstract])) OR (Nucleotide Polymorphisms, Single[Title/ Abstract])) OR (Polymorphisms, Single Nucleotide[Title/Abstract])) OR (Single Nucleotide Polymorphisms[Title/Abstract])) OR (SNPs[Title/Abstract])) OR (Single Nucleotide Polymorphism[Title/Abstract])) OR (rs[Title/Abstract])) AND (("Breast Neoplas m s " [Mesh]) OR

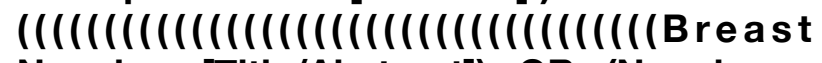
Neoplasm[Title/Abstract]) OR (Neoplasm, Breast[Title/Abstract])) OR (Breast Tumors[Title/Abstract])) OR (Breast Tumor[Title/Abstract])) OR (Tumor, Breast[Title/Abstract])) OR (Tumors, Breast[Title/Abstract])) OR (Neoplasms, Breast[Title/Abstract])) OR (Breast Cancer[Title/Abstract])) OR (Cancer, Breast[Title/Abstract])) OR (Mammary Cancer[Title/Abstract])) OR (Cancer, Mammary[Title/Abstract])) OR (Cancers, Mammary[Title/Abstract])) OR (Mammary Cancers[Title/Abstract])) OR (Malignant Neoplasm of Breast[Title/Abstract])) OR (Breast Malignant Neoplasm[Title/ Abstract])) OR (Breast Malignant Neoplasms[Title/Abstract])) OR (Malignant Tumor of Breast[Title/Abstract])) OR (Breast Malignant Tumor[Title/Abstract])) OR (Breast Malignant Tumors[Title/ Abstract])) OR (Cancer of Breast[Title/ Abstract])) OR (Cancer of the Breast[Title/ Abstract])) OR (Mammary Carcinoma, Human[Title/Abstract])) OR (Carcinoma,
Human Mammary[Title/Abstract])) OR (Carcinomas, Human Mammary[Title/ Abstract])) OR (Human Mammary Carcinomas[Title/Abstract])) OR (Mammary Carcinomas, Human[Title/Abstract])) OR (Human Mammary Carcinoma[Title/ Abstract])) OR (Mammary Neoplasms, Human[Title/Abstract])) OR (Human Mammary Neoplasm[Title/Abstract])) OR (Human Mammary Neoplasms[Title/ Abstract])) OR (Neoplasm, Human Mammary[Title/Abstract])) OR (Neoplasms, Human Mammary[Title/Abstract])) OR (Mammary Neoplasm, Human[Title/ Abstract])) OR (Breast Carcinoma[Title/ Abstract])) OR (Breast Carcinomas[Title/ Abstract])) OR (Carcinoma, Breast[Title/ Abstract])) OR (Carcinomas, Breast[Title/ Abstract]))).

Participant or population: Breast cancer patients.

Intervention: Single nucleotide polymorphisms associated with breast cancer risk.

Comparator: Healthy person.

Study designs to be included: Case-control study.

Eligibility criteria: Tumor group:1. Patients with breast cancer confirmed by histopathological studies2. There are no restrictions on menopause, gender, age, country or region3. There are no restrictions on breast cancer classification, staging, surgery or drug therapy4. Exclude duplicate reports, animal studies, and review studies; Studies in which SNPs demonstrated a departure from HardyWeinberg equilibrium (HWE) in controls were excluded.

Information sources: Pubmed and Embase databases.

Main outcome(s): A total of $\mathbf{3 7 0}$ articles were included in the meta-analysis. After direct meta-analysis, 31 genes were included in the network meta-analysis, and 7 relative genes were obtained: MTHFR 
RS1801131, Fasrs2234767, PHBrs6917, microRNArs 3746444 , II-8 rs4073, BRCA1rs8176318, APE1rs1760944. Thakkinstain's algorithm was used to obtain the optimal genetic model for the 7 genes. After comparison of FPRP results, the optimal gene APE1rs1760944 was obtained, and the optimal model was codominant model.

Quality assessment / Risk of bias analysis: Two reviewers will independently assess risk of bias based on the following domains from recommendations from the Cochrane handbook: 1. Adequate sequence generation; 2. Allocation concealment; 3. Blinding; 4. Incomplete outcome data and how it was addressed; 5. Selective reporting of the outcome.

Strategy of data synthesis: (1)whether to describe genotyping methods; (2)Whether to describe the population stratification method; (3)Whether to describe genotype inference method; (4) Whether the genotype distribution of the control group conforms to HWE; (5)Whether to emphasize the repeatability of research; (6)Whether to describe the inclusion and exclusion criteria and matching methods for the research objects; (7)Whether the statistical method and software version are explained; (8)Correlation judgment method; (9) Whether the data is sufficient.

Subgroup analysis: Due to the lack of data and the lack of repeatability, it is impossible to carry out further subgroup analysis of the data.

Sensitivity analysis: The exclusion of lowquality studies, the use of different statistical methods or models to analyze the same data after low sensitivity, exclusion did not have a significant impact on the results.

\section{Country(ies) involved: China.}

Keywords: Breast cancer, single nucleotide polymorphisms, network meta-analysis, FPRP.
Contributions of each author:

Author 1 - Ying yuan Wang. Email: wyy15596828515@163.com Author 2 - Ze chang Chen. Author 3 - Lu xin Zhang. Author 4 - Shuang yi Chen. 